\title{
Scientific Analysis of NPAR Processing of EBSD Results for Beam-Sensitive Materials
}

\author{
$\underline{\text { Patrick P. Camus }}^{1}$, Stuart I. Wright ${ }^{1}$, Matthew M. Nowell ${ }^{1}$, Rene de Kloe $^{2}$ \\ 1. EDAX Inc., 91 McKee Place, Mahwah, NJ 07430 USA \\ 2. Ametek BV, PO Box 4144, 5004JC Tilburg, The Netherlands
}

When collecting EBSD data, decisions are made by the operator to select the number of camera pixels in the diffraction pattern and balance the pattern quality with acquisition time. The first decision is usually selecting the number of camera pixels that are needed for the accurate indexing of the crystal structure: more complex crystal structures require more bands and pixels than elementary cubic systems. The highest quality patterns for a given beam current are obtained by increasing the exposure time and the number of averaged frames at the expense of increasing the collection times [1]. It should be noted that high quality patterns are those that produce high band detection; it is not necessary that the patterns look visually stunning. An additional constraint must be considered for beam-sensitive materials in that the desired beam current may damage or destroy the sample. If the decision has been made to reduce the collection time and therefore the pattern quality, band detection will begin to suffer, which will decrease indexing quality, producing lower quality mapping data. The decision needs to be made on how low an indexing quality is permissible to still obtain the needed analytical results. Correction methods must then be applied which can restore some, if not most, of the mapping data quality.

The usual method of increasing the quality of the mapping results is to apply kernel operations to the mapping data. Most of these kernel operations do not involve measuring the average value of the kernel pixels but involve a median filter to replace a value of the current "bad" pixel within the map data with data from a "good" neighboring pixel [2]. For isolated or infrequent "bad" pixels, these kernel operations work very well, however for lower index quality maps from beam-sensitive materials, these operations can have significant shortcomings.

NPARTM is a routine which can restore the quality to EBSD mapping analyses by increasing the quality of the patterns themselves [3]. The routine replaces the pixel diffraction pattern with the average of the patterns within a kernel, Figure 1, followed by traditional indexing routines. This has the effect of both reducing the noise and increasing the signal in the pattern for an enhanced indexing ability. The effect is similar to, but different from, camera frame averaging during acquisition, but at a significantly reduced acquisition time. Obviously the limitation of this routine is the need to have the neighborhood diffraction patterns available during the indexing operation. The most striking example of NPAR is when it is applied to a low-percentage indexed data set and provides mapping results with significantly higher indexed pixels. In maps with nearly perfect indexing results, the effects are much less obvious but can still be significant. It should be noted that all traditional data correction methods can be applied to the new NPARprocessed data, although this is rarely needed. The beneficial results of the NPAR routine on mapping data are well documented, but the detailed analysis of the changes performed on the data has not been presented. This paper looks into the detail of the changes that occur to data sets when applying ONLY the NPAR correction to high-quality and low-quality data sets.

An annealed nickel sample was used as a control. OIM maps were acquired using EDAX TEAM ${ }^{\mathrm{TM}}$ software with an $\sim 10 \mathrm{nA} 20 \mathrm{kV}$ field-emission beam for 186x150 pixels. The EDAX Hikari camera image was binned to $60 \times 60$ pixels with an exposure time of $0.94 \mathrm{~ms}$ for $1000 \mathrm{fps}$. An OIM map of a Brachiopod shell (Novocrania anomala) was collected for $1130 \times 916$ pixels using $\sim 1 \mathrm{nA}$ at $15 \mathrm{kV}$ with an acquisition rate of $\sim 35$ pps. The diffraction patterns were saved for each pixel as is necessary for processing by NPAR. 
The diffraction patterns in Figure 1 illustrate the increase in the pattern quality at the short exposure times using NPAR. For long exposure times, high quality OIM maps are observed using the raw data, Figure 1a, which does not improve significantly when using the NPAR processing, Figure $2 \mathrm{~b}$. A comparison of the individual pixel changes in a few OIM indexing metrics were performed, of which the IQ values are shown in Figure 2c. The low beam current necessary to preserve the sample integrity of the Brachiopod shell produces low quality OIM maps, Figure 3a, which are significantly improved by application of the NPAR routine, Figure 3b. A comparison of the individual pixel changes in the Fit values are shown in Figure 3c.

\section{References}

[1] Wright, S. and M. Nowell (2008). Advanced Materials and Processes. 166: 29-31.

[2] Wright, S. I. (2006). Materials Science and Technology 22(11): 1287-1296.

[3] Wright, S. I., M. M. Nowell, S. P. Lindeman, P. P. Camus, M. De Graef and M. A. Jackson (2015). Ultramicroscopy 159: 81-94.

[4] Specimen courtesy of E. Griesshaber, Geo- und Umweltwisschaften, Ludwig-Maximilians-Universität München

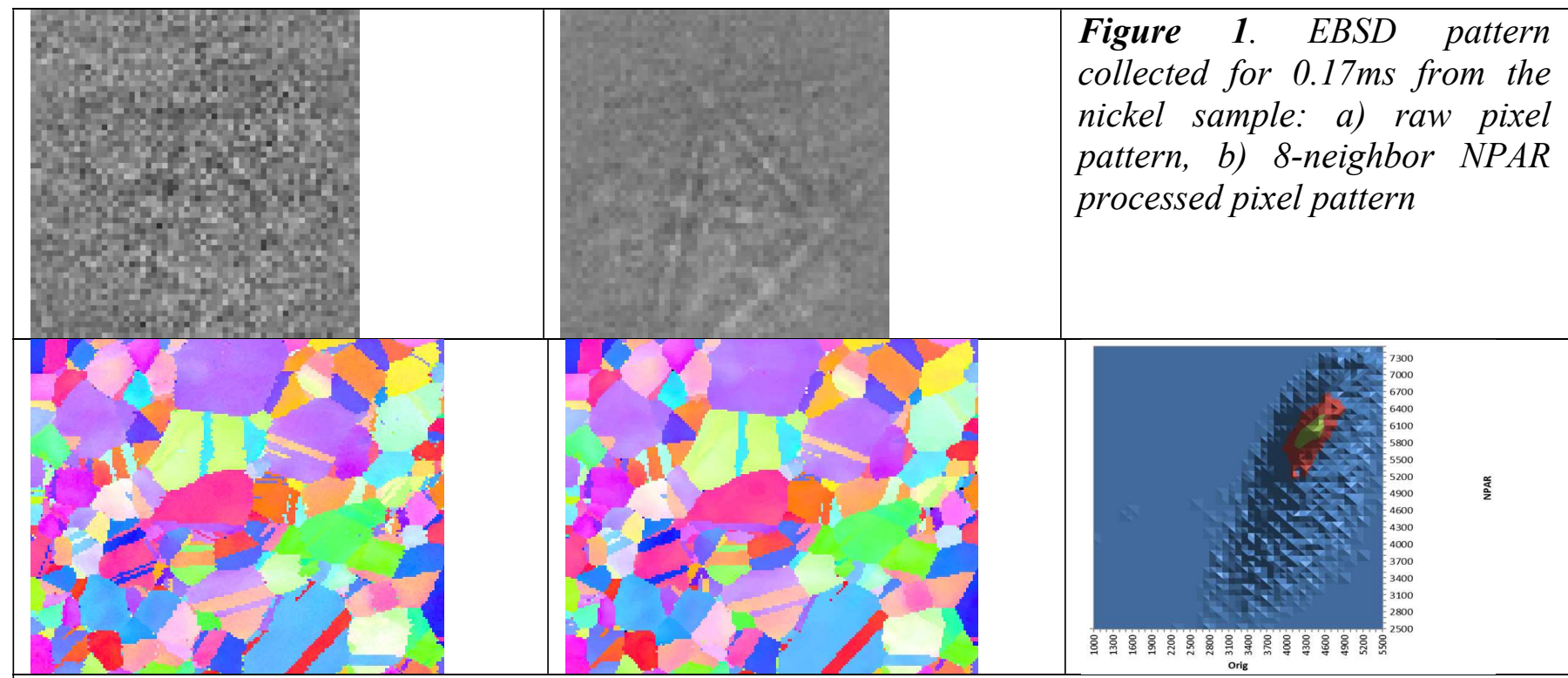

Figure 2. IPF maps of nickel show (a) high quality maps, (b) NPAR processing map which reduces the noise associated with boundary edges but also "removes" very thin twins and grains, and (c) a plot of the pixel IQ values before and after NPAR processing.

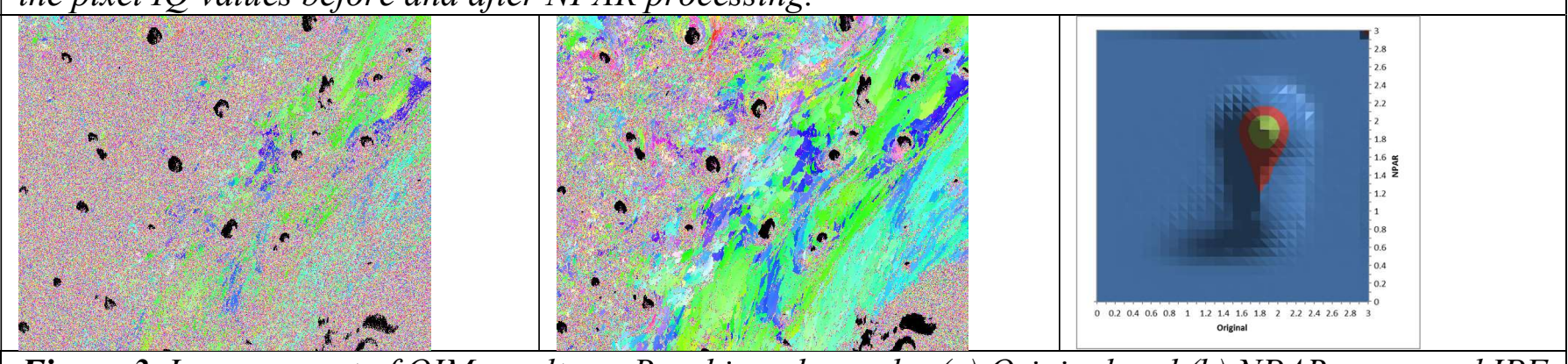

Figure 3. Improvement of OIM results on Brachiopod sample: (a) Original and (b) NPAR processed IPF maps and (c) a plot of the pixel Fit values before and after processing. 